\title{
Intermediate scales of symmetry breaking in Calabi-Yau models*
}

\author{
M. Masip \\ Institute for Fundamental Theory \\ Department of Physics, University of Florida \\ Gainesville, Florida 92611, USA
}

\section{ABSTRACT}

We discuss the generation of large intermediate scales of symmetry breaking in grand unification models suggested by the heterotic string. We analyze on dimensional grounds a particular scenario where one flat direction in the effective potential defines two different scales of gauge-symmetry breaking at very high energies $\left[\approx O\left(10^{15} \mathrm{GeV}\right)\right]$. This mechanism implies the presence of one light $[O(1 \mathrm{TeV})]$ nonchiral neutrino. The size of the observable low-energy effects seems, however, quite model depentent.

* Work supported by the DGICYT (MEC, Spairı). 


\section{INTRODUCTION}

The 10-dimensional $E_{8} \times E_{8}$ heterotic string ${ }^{1}$ contains near the Planck scale more dimensions, symmetry and matter than the observed at low energies. To obtain realistic models, it is necessary to assume that the (unknown) dynamics of the theory favours a vacuum that compactifies the six extra dimensions, breaks the excess of symmetry, and makes the extra fields heavy enough.

In the framework of compactification on a 3-generation Calabi-Yau manifold, ${ }^{2}$ one may distinguish several processes that decouple from the model its exotic ingredients. The identification of the spin connection of the manifold in the gauge fields leaves in the observable sector $N=1$ supersymmetry (SUSY) and $E_{6}$ gauge symmetry, with chiral superfields in the 27 and $\overline{27}$ representations of this group. In the known cases, the 3 -family manifold is constructed from a non-simply connected one, where non-trivial gauge configurations provide a breaking of the remaining $E_{6}$ symmetry to a smaller rank-six group. ${ }^{3}$ This process will introduce a convenient asymmetry (not present in the $E_{6}$ model) between the quark and lepton sectors. The effective theory must also break SUSY. In the most promising scenario, ${ }^{4}$ SUSY is broken at large scales in a sector connected just through gravitational interactions with quarks and leptons and one obtains the usual (order $1 \mathrm{TeV}$ and universal at the Planck scale) soft-breaking terms. Finally, in order to reduce the gauge symmetry to the rank-four group $S U(3)_{C} \times S U(2)_{L} \times U(1)_{Y}$, the evolution of the model down to low energies must define intermediate scales (IS) of symmetry breaking. Below these scales the exotic fields in the three chiral 27 of $E_{6}$ (two type down quarks, two neutrinos and two Higgs doublets) will combine into nonchiral representations of the Standard-Model symmetry and will (possibly) become massive. The vectorlike $27+\overline{27}$ multiplets will also acquire masses through effective nonrenormalizable interactions. The IS must be very large (typically $10^{15} \mathrm{GeV}$ ), since a slow proton decay and an acceptable value of the low-energy gauge couplings require that we are left at these energies with essentially a minimal supersymmetric extension of the Standard Model. ${ }^{7}$

Although the IS represent another source of uncertainty in the conexion of the string with its observable limit, it has been argued ${ }^{8}$ that they have model-independent implications that may be accesible to experiment. In particular, the IS would imply the existence of $O(1 \mathrm{TeV})$ fields producing neutrino masses and lepton-number violating processes. We would like here briefly review the mechanism that generates these IS and also introduce a particular structure in the superpotential $(P)$ with some interesting properties. This structure requires just one flat direction to produce the two stages of symmetry breaking required in rank-six models. It provides differences of several orders of magnitude between the IS. In a realistic low-energy model, the Higgs sector (whose lightness is not protected by chirality ${ }^{7}$ ) and the neutrino sector (where see-saw masses ${ }^{5}$ would reflect the 
physics in the desert) are in principle sensitive to the splitting of the scales. This particular structure is not contained in the analysis done by Arnowitt and Nath, ${ }^{8}$ where two independent flat directions are implictly assumed. In contrast with them, we find only one nonchiral neutrino necessarily light $[O(1 \mathrm{TeV})]$ and other quite model-dependent observable effects.

When we need to be definite we will refer to the Tian-Yau 3-generation manifold ${ }^{6-14}$ with

$$
S U(3)_{C} \times S U(3)_{L} \times S U(3)_{R} \subset E_{6}
$$

gauge symmetry, which is the first and most extensively studied case. However, our arguments wiil also be relevant for the other 3-generation models (Schimmrigk and bicubic in $P^{2} \times P^{2}{ }^{15}$ ). In the Tian-Yau model the non-singlet-gauge matter consists of nine families of leptons $\lambda$, six of $\bar{\lambda}$, seven of quarks $q$ and antiquarks $Q$, and four of $\bar{q}$ and $\bar{Q}$, where

$$
27 \longrightarrow \lambda \equiv(1, \overline{3}, 3)+q \equiv(3,3,1)+Q \equiv(\overline{3}, 1, \overline{3})
$$

and the assignment of standard quantum numbers is

$$
\lambda \sim\left(\begin{array}{ccc}
h^{0} & h^{\prime-} & e \\
h^{+} & h^{\prime 0} & \nu \\
e^{c} & \nu_{4} & \nu_{5}
\end{array}\right) ; q \sim\left(\begin{array}{cc}
u & \\
d & \times 3 \text { colours } \\
d^{\prime} &
\end{array}\right) ; Q \sim\left(\begin{array}{cc}
u^{c} & \\
d^{c} & \times 3 \text { colours } \\
d^{\prime c} &
\end{array}\right) .
$$

\section{GENERATION OF INTERMEDIATE SCALES}

Our starting point is the effective model at the compactification/unification scale $M_{c}\left(<M_{P l a n c k}\right)$. We suppose that the residual $N=1$ SUSY is broken in the effective potential by scalar masses and by a term proportional to $P$. The SUSY-breaking effects would also fix the vacuum expectation values (VEV's) of the moduli fields (whose potential is in principle flat). These VEV's determine the complex structure of the manifold and then the value of all the couplings in $P$. We will assume that $P$ incorporates a group of discrete symmetries acting in a definite way over each multiplet of chiral superfields. In Calabi-Yau models these symmetries are necessary to achieve the hierarchies $M_{Z} / M_{c}$ or $i n_{u p} / m_{\text {top }},{ }^{9}$ and also to define a low-energy matter parity. ${ }^{10}$

Since the supersymmetric part of the effective potential is positive semidefinite, a nontrivial minimum should be favoured by the SUSY-breaking terms. Although these effective terms are in principle flavour independent (they are generated via gravitational interactions), their universality is broken by Yukawa radiative corrections. In Ref. 11 is showed via the renormalization group that the mass coefficients evolve fast (in one or two orders of magnitude) to negative values when one scales down tize effective model, triggering nonzero VEVs. Then, if the potential contains a flat direction (a direction in the field space without self-interactions) the VEVs will grow up to values as large as the 
scale where these mass coefficients become negative. It is remarkable that the Tian-Yau model mentioned above contains more families and couplings of leptons $\lambda$ than of quarks $q$ and $Q$, what favours VEV's for fields in lepton multiplets and preserves the colour symmetry. (A larger number of $\lambda$ s above the IS is also necessary for a correct unification, since the gauge couplings verify $\alpha_{C}>\alpha_{L, R}$.)

The flatness of the potential is a necessary ingredient to define large IS. The supersymmetric scalar potential includes the $D$ and $F$ terms:

$$
\begin{gathered}
D^{\alpha}=g \phi_{i}^{\dagger} T_{j}^{\alpha i} \phi^{j}, \quad F_{\Phi_{i}}=\frac{\partial P}{\partial \Phi_{i}}, \\
V(\phi)=\sum_{i}\left|F_{\Phi_{i}}\right|^{2}+\sum_{\alpha}\left|D^{\alpha}\right|^{2} .
\end{gathered}
$$

$D$-flatness is obtained by requiring that for each VEV along a gauge flavour there is an identical VEV along a field in the conjugate representation; since these fields have opposite charges, their $D$-contributions cancel. $F$-flatness is obtained if all the terms in $P$ contain more than one field with zero VEV. When only $\nu_{5}$ and $\nu_{4}$ [see (1.3)] develop VEV's, the gauge symmetry provides flatness with respect to trilinears in $P$. However, including effective $(27 \overline{27})^{n}$ nonrenormalizable terms (which we assime suppressed by inverse powers of $M_{c}$ ), the nonflat contributions can be forbidden only with the help of the discrete symmetries of the model.

In general, it will be difficult to find models with completely flat directions. Suppose that the VEV's along $\nu_{5}$ involves $n$ families; there are $n+1$ equations $\left(D=0\right.$ and $F_{i}=0$, with $i=1, \ldots, n)$ but just $n$ VEV's, and then a flat direction would require two dependent equations. The case with only one dependent equation (like in Ref. 12) corresponds to a discrete set of zeroes, with cancellations between terms of different dimension that would imply VEV's order $M_{c}$. Actually, completely flat directions have been found only in models that incorporate an $R$-symmetry. ${ }^{13,14}$ Experience shows, however, that when flatness is protected by an unbroken symmetry one also gets a large amount of extra massless fields, which tend to move the electroweak angle and the proton lifetime to non-acceptable values (this is the case, for instance, in Ref. 13).

Another possibility, easier to realize in models without $R$-symmetries, is the generation of IS along non-completely flat directions. If flatness is broken just by terms of high dimension (very suppressed by powers of $\mathrm{H}_{c}^{-1}$ ), the scales may be large enough. Suppose that the effective potential receive contributions from terms $(27 \overline{27})^{n}$ in $P$. On dimensional grounds, the minimum will result from the balance between order $m_{s}^{2} \approx 1 \mathrm{TeV}^{2}$ (negative) biliniars and order $M_{c}^{-4 n+6}$ (positive) $F$-contributions of dimension $4 n-2$. The VEV's, order ${ }^{2 n-2} \sqrt{m_{s} / M_{c}} M_{c}$, will be one or two orders of magnitude below $M_{c}$ (as required by perturbative unification of the gauge coplings) for $n=4-6$. (The soft 
contribution proportional to $P$, order $m_{s} . M_{c}^{2 n-3}$, is unnecessary in this argument, because it is not the dominant for any value of the fields.)

We note that in the breaking along an almost flat direction the nonzero value of the $F$ terms introduce a new source of SUSY breaking that will involve the scalars interacting with the fields developing VEV's. Contrarily to the ones coming from gravitational in.teractions, these soft-breaking terms are not universal and do not affect to the three chiral families (massless after the IS), although they could affect to the Higgs fields. We also observe that the fermionic partners of the scalars developing a VEV (non-weakly interacting neutrinos) receive only order $m_{s}$ mass contributions from the terms $(27 \overline{27})^{n}$ in $P$. However, the Yukawa couplings of these neutrinos with the low-energy fields are necesarily very small (order $m_{s} /$ IS $\approx 10^{-12}$ ); sizeable trilinears [of type $\nu_{5}\left(h^{0} h^{+}\right)\left(h^{\prime-} h^{\prime 0}\right)$ ] in $P$ would give large masses to the low-energy fields.

Finally, we would like to mention the cosmological scenario that may accommodate this type of phase transitions. ${ }^{12,19}$ The finite-temperature effective potential would contain a quadralic term proportional to $T^{2}$, where $T$ is the temperature of the thermal bath in contact with the scalar field. For the field with flat potential that we are considering, this term prevents the transition (from zero to large VEV's) untill the temperature of the Universe is $C\left(m_{s} \approx 1 \mathrm{TeV}\right)$. Then, the entropy generated would dilute to non-acceptable values any existing baryon asymmetry. There are several ways to evoid this problem: to suppose a period of inflation (where the Universe is extremely cool before reheating and baryogenesis); to recreate the baryon number at $T=O(1 \mathrm{TeV})$ (trasferring energy to heavy fields via anharmonic couplings); or simply to assume that the initial values of the field [at $T=O\left(M_{c}\right)$ ] are likely to be large (and the field would be trapped when $T$ drops below the IS).

\section{A MODEL WITH TWO INTERMEDIATE SCALES}

The rank-six models under study here require two IS of gauge-symmetry breaking, defined by VEV's of two independent combinations of $\nu_{4}$ and $\nu_{5}$ (with identical $\vec{\nu}_{4}$ and $\bar{\nu}_{5}$ VEV's) in two different $\lambda+\bar{\lambda}$ vectorlike multiplets. It will be possible to make a gauge transformation and leave the multiplet containing bigger VEV's with $\left\langle{ }^{1} \nu_{4}\right\rangle=0$ (the superindexs label the two $\lambda$ and $\bar{\lambda}$ ). The smaller VEV's in the second family will in principle involve both gauge flavours. However, the fields ${ }^{2} \nu_{5}$ and ${ }^{2} \bar{\nu}_{5}$ are not neutral respect the possible matter parities one can define in the Tian-Yau manifold (see Table II). Since a low-energy matter parity is a necessary ingredient of any realistic SUSY model, these fields should not develop VEV's. We will consider models where this possibility is favoured by the discrete symmetries, and the VEV's in ${ }^{1,2} \lambda$ and ${ }^{1,2} \bar{\lambda}$ satisfy:

$$
\begin{aligned}
& \left.\left\langle{ }^{1} \nu_{5}\right\rangle=\left\langle{ }^{1} \overline{\nu_{5}}\right\rangle=\phi_{1}, \quad\left\langle{ }^{1} \nu_{4}\right\rangle=<{ }^{1} \overline{\nu_{4}}\right\rangle=0 ; \\
& \left.\left.\left\langle{ }^{2} \nu_{5}\right\rangle=<{ }^{2} \overline{\nu_{5}}\right\rangle=0, \quad\left\langle{ }^{2} \nu_{4}\right\rangle=<{ }^{2} \overline{\nu_{4}}\right\rangle=\phi_{2},
\end{aligned}
$$


We define $x \equiv \phi_{1} / M_{c}$ and $y \equiv \phi_{2} / M_{c}(1>x>y)$.

In the Tian-Yau model with $S U(3)_{C} \times S U(3)_{L} \times S U(3)_{R}$ gauge symmetry, the first VEV's [in Eq. (3.1a)] break the symmetry to $S U(3)_{C} \times S U(2)_{L} \times S U(2)_{R} \times U(1)_{B-L}$, nine gauginos combine with the fermions ${ }^{1} e,{ }^{1} \bar{e},{ }^{1} e^{c},{ }^{1} \bar{e}^{c},{ }^{1} \nu,{ }^{1} \bar{\nu},{ }^{1} \nu_{4},{ }^{1} \bar{\nu}_{4}$, and $\frac{1}{\sqrt{2}}\left({ }^{1} \nu_{5}-{ }^{1} \bar{\nu}_{5}\right)$ in the first supermultiplets, defining Dirac fields. At the second scale $\left[\phi_{2}\right.$ in (3.1b)] other three gauginos combine with ${ }^{2} e^{c},{ }^{2} \bar{e}^{c}$ and $\frac{1}{\sqrt{2}}\left({ }^{2} \nu_{5}-{ }^{2} \bar{\nu}_{5}\right)$ leaving unbroken just the Standard-Model symmetry, $S U(3)_{C} \times S U(2)_{L} \times U(1)_{Y}$.

In principle, the $\nu_{5}$ and $\nu_{4}$ VEV's in Eq. (3.1) should grow along two independent flat directions of the scalar potential. We are going to discuss a different and more economic scenario where just one almost flat direction is sufficient to generate the two IS. This is achieved for a specific form of the ${ }^{1} \nu_{5}-{ }^{2} \nu_{4}$ interactions that will fix a small $\left\langle{ }^{2} \nu_{4}\right\rangle$ component when $\left\langle{ }^{1} \nu_{5}\right\rangle$ grows along the flat direction and will define a hierarchy of type $y=O\left(x^{a}\right)$.

To discuss the orders of magnitude, we will consider the model with superpotential

$$
P=\frac{\alpha}{M_{c}^{2 n-3}}\left({ }^{1} \nu_{5}{ }^{1} \bar{\nu}_{5}\right)^{n}+\frac{\beta}{M_{c}^{2 m-3}}\left({ }^{2} \nu_{4}{ }^{2} \bar{\nu}_{4}\right)^{m}+\frac{\gamma}{M_{c}^{2(i+j)-3}}\left({ }^{1} \nu_{5}{ }^{1} \bar{\nu}_{5}\right)^{i}\left({ }^{2} \nu_{4}{ }^{2} \bar{\nu}_{4}\right)^{j}
$$

and effective scalar masses destabilizing the potential along ${ }^{1} \nu_{5}$ (i.e., $m_{1}^{2}{ }_{\nu_{5}}+m_{1_{\bar{\nu}_{5}}}^{2} \equiv$ $-m_{s}^{2}<0$ ). The actual value of the exponents in Eq. (3.2) would be fixed by the discrete symmetries of the particular manifold, while the parameters $\alpha, \beta$ and $\gamma$ are arbitrary complex coefficients that we assume order 1 . As shown in the previous section, if the ${ }^{1} \nu_{5}$ self-interactions (first term in $P$ ) define an almost flat direction $[n=4-6$ in Eq. (3.2)] these fields will develop large VEV's $\left\langle{ }^{1} \nu_{5}\right\rangle=\left\langle{ }^{1} \bar{\nu}_{5}\right\rangle=x M_{c}$, with $F$ contributions in the effective potential given by

$$
\begin{gathered}
F_{1_{\nu_{5}}}=F_{1 \bar{\nu}_{5}}=n \alpha M_{c}^{2} x^{2 n-1} \approx m_{s} M_{c} x, \\
F_{\nu_{\nu_{4}}}=F_{\bar{\nu}_{\bar{\nu}_{4}}}=0 .
\end{gathered}
$$

Now, we study the possibility of VEV's along the nonflat $[m=2-3 \text { in }(3.2)]^{2} \nu_{4}$ direction. If $\left.\left\langle{ }^{2} \nu_{4}\right\rangle=<^{2} \bar{\nu}_{4}\right\rangle=y M_{c}$, the $F$ contributions become

$$
\begin{gathered}
F_{\nu_{\mathrm{s}}}=F_{\bar{\nu}_{\mathrm{s}}}=n \alpha M_{c}^{2} x^{2 n-1}+i \gamma M_{c}^{2} x^{2 i-1} y^{2 j}, \\
F_{2_{\nu_{4}}}=F_{\overline{2}_{\bar{\nu}_{4}}}=m \beta M_{c}^{2} y^{2 m-1}+j \gamma M_{c}^{2} x^{2 i} y^{2 j-1} .
\end{gathered}
$$

The dimensionally less suppressed contribution is the first term in $F_{2} \nu_{4}$. We find, however, that if the discrete symmetries of the model fix the exponents in (3.2) such that

$$
i+\frac{n}{m} j=n
$$


there will be a value of $\phi_{2}$ (small respect to $\phi_{1}$ ) that exactly cancels the nonflat contributions in $F_{2} \nu_{4}$ while adding to $F_{\nu_{5}}$ just almost flat contributions (of the same order as the one introduced by $\left.\phi_{1}\right)$. This value of $\phi_{2}\left(=y M_{c}\right)$ is

$$
y=\left(\frac{-j \gamma}{m \beta}\right)^{\frac{1}{2(m-j)}} x^{\frac{n}{m}}
$$

and the $F_{\nu_{\mathrm{B}}}$ term fixing the minimum becomes

$$
F_{\nu_{5}}=\left[n \alpha+i \gamma\left(\frac{-j \gamma}{m \beta}\right)^{\frac{j}{(m-j)}}\right] M_{c}^{2} x^{2 n-1}
$$

Depending on the specific value of $\alpha, \beta$ and $\gamma$ but without need of fine tunning this combination of two terns in $F_{\nu_{\mathrm{s}}}$ may be more flat than the direction with $\phi_{2}=0$, and then may correspond to a deeper minimum. Although both minima will be of the same order $\left[O\left(\left|F_{1} \nu_{s}\right|^{2}\right)\right]$, they are separated (when $\phi_{2}$ varies from zero to the value that cancels $\left.F_{2} \nu_{4}\right)$ by the barrier $\left|F_{2} \nu_{4}\right|^{2}$ in Eq. (3.4b).

We should emphasize that the ingredients to generate such a minimum [a flat direction favoured by the effective masses and interacting with another direction as specified in Eq. (3.5)] are not rare in Calabi-Yau models, where tipically one is left at $M_{c}$ with many different families and large groups of discrete symmetries. In Ref. 9, for example, is singled out a model (unique within its class) that satisfies certain phenomenological requirements. We find that in this case the IS have to be generated through the discussed mechanism, with $n=6, m=2, i=3$ and $j=1$; for $m_{s}=1 \mathrm{TeV}$ and $M_{c}=10^{16.5} \mathrm{GeV}$ we obtain

$$
\phi_{1}=O\left(\sqrt{\frac{m_{s}}{M_{c}}}\right) M_{c} \approx 10^{15.2} \mathrm{GeV} ; \quad \phi_{2}=O\left(x^{3}\right) M_{c} \approx 10^{12.5} \mathrm{GeV} .
$$

Other possibilities are tabulated in Table I.

\section{LOW ENERGY IMPLICATIONS}

As pointed out by Arnowitt and Nath, ${ }^{8}$ the flatness arguments have implications over the low-energy matter content. In particular, they found that in the Tian-Yau model the symmetry breaking along two almost flat directions leaves a minimum of two (and possibly four) nonchiral neutrinos with light masses (order $m_{s}$ ). We are going to show that our way of generating the IS has different phenomenological implications.

The nonchiral neutrinos in the two vectorlike $\lambda+\bar{\lambda}$ multiplets will receive mass contributions from gauge and chiral interactions. The first type of interactions will combine them with the gauginos associated to the lost symmetries, as stated in the previous section. Chiral interactions will affect in a different way to the fields involved in each of 
the IS. While ${ }^{1} \nu_{5},{ }^{1} \bar{\nu}_{5},{ }^{1} \nu_{4}$ and ${ }^{1} \bar{\nu}_{4}$ will receive from terms $\left({ }^{1} \lambda^{1} \bar{\lambda}\right)^{n}$ in $P$ order $1 \mathrm{TeV}$ masses, the neutrinos in the second family will receive from $\left({ }^{2} \lambda^{2} \bar{\lambda}\right)^{m}$ and $\left({ }^{1} \lambda^{1} \bar{\lambda}\right)^{i}\left({ }^{2} \lambda^{2} \bar{\lambda}\right)^{j}$ heavy mass contributions [in the case corresponding to (3.8), the masses come from terms $\left({ }^{2} \lambda^{2} \bar{\lambda}\right)^{2}$ and are order $\left.y^{2} M_{c} \approx 10^{9} \mathrm{GeV}\right]$. Considering all these mass contributions we obtain

a. one neutrino with mass $m_{1}=O(1 \mathrm{TeV}): \frac{1}{\sqrt{2}}\left({ }^{1} \nu_{5}+{ }^{1} \bar{\nu}_{5}\right) \equiv \nu_{1}^{c}$,

b. three neutrinos with intermediate mass $m_{2}\left[m_{2}=O\left(10^{9} \mathrm{GeV}\right)\right.$ for the case in (3.8)]: $\frac{1}{\sqrt{2}}\left({ }^{2} \nu_{4}+{ }^{2} \bar{\nu}_{4}\right) \equiv \nu_{2}^{c},\left[{ }^{2} \nu_{5}+O(y / x)^{1} \bar{\nu}_{4}\right] \equiv \nu_{3}^{c}$ and $\left[{ }^{2} \bar{\nu}_{5}+O(y / x)^{1} \nu_{4}\right] \equiv \nu_{4}^{c}$.

The neutrinos whose scalar superpartner develop a VEV can not get further masses without spoiling the flatness arguments. For this reason $\nu_{1}^{c}$ is necessarily light; if the flatness were exact, it would be massless. Although the presence of $\nu_{1}^{c}$ below $1 \mathrm{TeV}$ is a model independent feature, we find that definite predictions over its observable effects would require a specific model. This neutrino does not couple in $P$ with the rest of low-energy fields (see Section II). However, relevant couplings may appear in the gauge sector. After the first stage of breaking the gauginos $\left(\lambda^{-} \lambda^{0}\right)_{L}$ mix with the three chiral lepton doublets $(e \nu)_{i}(i=1, \ldots, 3)$, through mass terms of type

$$
\left\langle{ }^{1} \bar{\nu}_{5}{ }^{\dagger}>\left(\begin{array}{c}
1 \bar{e} \\
{ }^{1} \bar{\nu}
\end{array}\right)\left(\begin{array}{l}
\lambda^{-} \\
\lambda^{0}
\end{array}\right)_{L} ;\left\langle<\left({ }^{1} \nu_{5}{ }^{1} \bar{\nu}_{5}\right)^{a}>\left(\begin{array}{l}
{ }^{1} \bar{e} \\
{ }^{1} \bar{\nu}
\end{array}\right)\left(\begin{array}{l}
e \\
\nu
\end{array}\right)_{i} .\right.\right.
$$

Then, the gauge interactions of these gauginos with $\nu_{1}^{c}$ generate Yukawa couplings of type $\left({ }^{1} \tilde{e}^{1} \tilde{\nu}\right)^{\dagger} \overline{\nu_{1}^{c}}(e \nu)_{i}$. The sleptons $\left({ }^{1} \tilde{e}^{1} \tilde{\nu}\right)$ will have an order $\phi_{2} / \phi_{1} \equiv \sin \theta$ component along $\left({ }^{2} \tilde{h}^{\prime-2} \tilde{h}^{\prime 0}\right)$, which may have sizeable components along the space of the three low-energy sleptons. It results

$$
L_{Y}=\lambda_{i j} \sin \theta\left(\begin{array}{l}
\tilde{e} \\
\tilde{\nu}
\end{array}\right)_{i}^{\dagger} \overline{\nu_{1}^{c}}\left(\begin{array}{l}
e \\
\nu
\end{array}\right)_{j}+\text { h.c. }
$$

All the mixings are allowed by the $Z_{2}$ and $Z_{3}$ matter parities ${ }^{10}$ defined from discrete symmetries of the Tian-Yau manifold (see Table II). The Yukawas in Eq. (4.2) would induce at one-loop level effective operators of type anomalous magnetic moment $\left(l_{i}\right.$, with $i=1,2$ and 3 , stands for the charged leptons $e, \mu$ and $\tau$ )

$$
L_{e f f}=\frac{e}{4 m_{j}} F^{\mu \nu} \overline{l_{i}} \sigma_{\mu \nu}\left(a_{L}^{i j} P_{L}+a_{R}^{i j} P_{R}\right) l_{j}+\text { h.c. }
$$

producing lepton-number violation. For instance, in the process $\mu \longrightarrow \gamma e$ one obtains an amplitude proportional to $m_{\mu} / \bar{m}^{2}$, where $\bar{m}=O\left(m_{\text {slepton }}+m_{1}\right)$, with $a_{L}=\left(m_{e} / m_{\mu}\right) a_{R}$ and then an emitted electron almost $100 \%$ left-handed polarized. Although this is an interesting effect, very constrained experimentally $\left[\left|a_{L}^{12}\right|+\left|a_{R}^{12}\right| \leq O\left(10^{-13}\right)\right]^{16,17}$, its size here depends on ratios of nonrenormalizable terms and appears to be, in consequence, model dependent. 
The fields in the intermediate region $m_{2}$ could be relevant for the masses of the three chiral neutrinos $\nu_{i}$. Since the scalars ${ }^{2} \nu_{5}$ and ${ }^{2} \bar{\nu}_{5}$ do not develop VEV's, there could be sizeable couplings in $P$ of type $h^{0} \nu_{i} \nu_{3,4}^{c}$ inducing see-saw neutrino masses. (Note that trilinears of $\nu_{3,4}^{c}$ with the two Higgs doublets are forbidden by the matter parities in Table II.) The heavy fields $\nu_{3,4}^{c}$ could make massive through this mechanism two of the three chiral neutrinos. Besides, one expects the couplings of $\nu_{4}^{c}$ (mainly in a $\overline{27}$ of $\left.E_{6}\right)$ to be smaller than those of $\nu_{3}^{c}$, what suggests one chiral neutrino $\left(\nu_{h}\right)$ heavier than the other two. In this scenario, cosmological constraints ${ }^{18}$ imply $m_{\nu_{h}}<O(100 \mathrm{eV})$. Assuming typical leptonic couplings, $\left[O\left(m_{\tau} /<h^{0}>\right)\right]$ this translates into a condition for the heavy neutrinos, $m_{z}>O\left(m_{\tau}^{2} / 100 \mathrm{eV}\right)=O\left(10^{7} \mathrm{GeV}\right)$, which is fulfilled just in some of the I rodels in Table I. It is possible, however, to make $\nu_{3,4}^{c}$ heavier and/or to suppress their couplings with $\nu_{i}$. Further masses for $\nu_{3,4}^{c}$ would arise through their combination with other neutrinos in 27 and $\overline{27}$ multiplets and with the $E_{6}$ singlets present in CalabiYau models. These mass terms would not spoil the flatness in the scalar potential (as it would happen with masses for $\nu_{1}^{c}$ and $\nu_{2}^{c}$ ). The second possibility (suppression of the couplings) is also quite model dependent. Actually, the $Z_{3}$ matter parity forbids this simplified scheme of see-saw masses. In this case, after diagonalizing the $5 \times 5$ mass matrix corresponding to $\nu_{i}, \nu_{3}^{c}$ and $\nu_{4}^{c}$, we find two heavy neutrinos $\left[\nu_{4}^{c}\right.$ and $\nu_{3}^{c}+$ $\left.O\left(m_{\tau} / m_{2}\right) \nu_{i}\right]$ defining a Dirac field plus three massless neutrinos $\left[\nu_{i}+O\left(m_{\tau} / m_{2}\right) \nu_{3}^{c}\right]$. For these reasons, although the scenario suggests masses for the Standard Model neutrinos, even the estimation of orders of magnitude precises a more specific model.

\section{CONCLUSIONS}

We have analyzed a mechanism that requires just one almost flat direction in the scalar potential to generate the two stages of gauge-symmetry breaking precised in ranksix Calabi-Yau models. This nechanism is not contained in the general analysis done by Arnowitt and Nath (where two flat directions are assummed), and consequently its implications are different from those. We find the second scale of breaking several orders of magnitude smaller than the first. We also find only one nonchiral neutrino (the one along the flat direction) necessarily light, with mass contributions in the intermediate region for the neutrinos in the multiplet defining the second scale. This scenario has non-Standard low-energy implications, such as see-saw masses for the chiral neutrino: and lepton-number violating processes. However, their size appears to be quite model dependent. An analysis of an explicit model where this mechanism is realised will be done elsewhere.

\section{ACKNOWLEDGMENTS.}

The author wish to thank J. Hong for discussions, P. Ramond for discussions and a reading of the manuscript, and the IFT for the hospitality extended to him. He also 
aknowledges a FPI fellowship from the Ministerio de Educación y Ciencia (Spain).

\section{References}

1 D. Gross, J. Harvey, E. Martinec and R. Rohmı, Phys. Rev. Lett. 55 (1985) 502; Nucl. Phys. B 256 (1985) 253; M. Green, J. Schwarz and E. Witten, Superstring Theory (Cambridge University Press, 1987).

${ }^{2}$ S.T. Yau, in Simposium on Anomalies, Geometry and Topology, ed. W.A. Bardeen and A.R. White (World Scientific, Singapore, 1985).

${ }^{3}$ Y. Hosotani, Phys. Lett. 126 B (1983) 309; E. Witten, Nucl. Phys. B 258 (1985) 75.

${ }^{4}$ H.P. Nilles, Phys. Lett. 115 B (1982) 193; M. Dine, R. Rohm, N. Seiberg and E. Witten, Phys. Lett. 156 B (1985) 55.

${ }^{5}$ J. Harvey, D. Reiss and P. Ramond, Nucl. Phys. B 119 (1982) 223.

${ }^{6}$ B.R. Green, K.F. Kirklin, P. Miron and G.G. Ross, Phys. Lett. B 180 (1986) 69; Nucl. Phys. B 278 (1986) 667; Nucl. Phys. B 292 (1987) 606; S. Kalara and R.N. Mohapatra, Phys. Rev. D 35 (1987) 3143; R. Arnowitt and P. Nath, Phys. Rev. Lett. 60 (1988) 1817; G. Lazarides and Q. Shafi, J. Math. Phys. 30 (1989); P. Berglund, T. Hubsch and L. Parkes, preprint HUTMP-90/B298.

${ }^{7}$ F. del Aguila, G.D. Coughlan and M. Masip, Phys. Lett. 227 B (1989) 55; Nucl. Phys. B 351 (1991) 90.

${ }^{8}$ R. Arnowitt and P. Nath, Phys. Rev. D 40 (1989) 191; Phys. Lett. B 244 (1990) 203; Phys. Rev. D 42 (1990) 2948; Phys. Rev. Lett. 66 (1991) 2708.

${ }^{9}$ F. del Aguila and M. Masip, preprint UG-FT-6/91.

${ }^{10}$ M.C. Bento, L. Hall and G.G. Ross, Nucl. Phys. B 292 (1987) 400; G. Lazarides and Q. Shafi, Nucl. Phys. B 338 (1990) 442.

${ }^{11}$ F. del Aguila and G.D. Coughlan, Phys. Lett. B 215 (1988) $\cong 3$.

12 G.G. Ross, lectures given at 1988 Banf Summer Institute, preprint CERN-TH-5109/88.

13 G. Lazarides, P.K. Mohapatra, C. Panagiotakopoulos and Q. Shafi, Nucl. Phys. B 233 (1989) 614.

${ }^{14}$ P. Berglund and T. Hubsch, Phys. Lett. B 260 (1991) 32.

15 M.G. Eastwood and T. Hubsch, Commun. Math. Phys. 132 (1990) 383.

${ }^{16}$ B.A Campbell, K. Enqvist, M.K. Gaillard, D.V. Nanopoulos, Int. J. Mod. Phys. A 2 (1987) 831.

${ }^{17}$ Pa Book, Review of Particle Properties, Phys. Lett. 239 B (1990) 1.

$\therefore$ G. Gelmini, S. Nussinov and R.D. Peccei, UCLA-91-TEP-15.

1' T. Ellis, K. Enqvist and D.V. Nanopoulos, Phys. Lett. 188 B (1987) 415; G. Lazarides, S. Panagiotakopoulos and Q. Shafi, Phys. Rev. Lett. 56 (1986) 557; O. Bertolami and G.G. Ross, Phys. Lett. 183 B (1987) 163. 
Table I. Order of magnitude of the IS and the masses of the neutrinos in the multiplet defining $\phi_{2}$.

\begin{tabular}{cccccc}
\hline$n$ & $\phi_{1} / M_{c}$ & $m$ & $\phi_{2} / M_{c}$ & $(i, j)$ & $m_{2}(\mathrm{GeV})$ \\
\hline 6 & $10^{-1.3}$ & 2 & $10^{-4.0}$ & $(3,1)$ & $10^{9}$ \\
& & 3 & $10^{-2.7}$ & $(2,2)$ & $10^{7}$ \\
& & 3 & $10^{-2.7}$ & $(4,1)$ & $10^{7}$ \\
& & 4 & $10^{-2.0}$ & $(3,2)$ & $10^{5}$ \\
\hline 4 & $10^{-2.7}$ & 2 & $10^{-5.4}$ & $(2,1)$ & $10^{6}$ \\
\hline
\end{tabular}

Table II. $Z_{2}$ and $Z_{3}$ matter parities defined from the discrete symmetries of the Tian-Yau manifold. ${ }^{10}$

\begin{tabular}{cccccc}
\hline Field & $\Xi_{2}$ & $Z_{3}$ & Field & $Z_{2}$ & $Z_{3}$ \\
\hline$(e \nu)_{i}$ & -1 & 1 & $\left.\left\langle{ }^{1} \nu_{5}\right\rangle,<{ }^{1} \bar{\nu}_{5}\right\rangle$ & +1 & 1 \\
$e^{c}{ }_{i}$ & -1 & $\alpha^{2}$ & $\left.\left\langle{ }^{2} \nu_{4}\right\rangle,<{ }^{2} \bar{\nu}_{4}\right\rangle$ & +1 & 1 \\
$(u d)_{i}$ & -1 & 1 & ${ }^{1} \nu_{4}$ & -1 & $\alpha$ \\
$u^{c}{ }_{i}$ & -1 & $\alpha$ & ${ }^{1} \bar{\nu}_{4}$ & -1 & $\alpha^{2}$ \\
$d^{c}{ }_{i}$ & -1 & $\alpha^{2}$ & ${ }^{2} \nu_{5}$ & -1 & $\alpha^{2}$ \\
$\left(h^{0} h^{+}\right)$ & +1 & $\alpha^{2}$ & ${ }^{2} \bar{\nu}_{5}$ & -1 & $\alpha$ \\
$\left(h^{\prime}-h^{\prime 0}\right)$ & +1 & $\alpha$ & $\left({ }^{1} e{ }^{1} \nu\right), \overline{\left({ }^{1} e{ }^{1} \nu\right)}$ & -1 & 1 \\
$\nu_{1}^{c}$ & +1 & 1 & $\left({ }^{2} h^{\prime-}{ }^{2} h^{10}\right), \overline{\left({ }^{2} h^{1-}-2 h^{10}\right)}$ & -1 & 1 \\
\hline
\end{tabular}



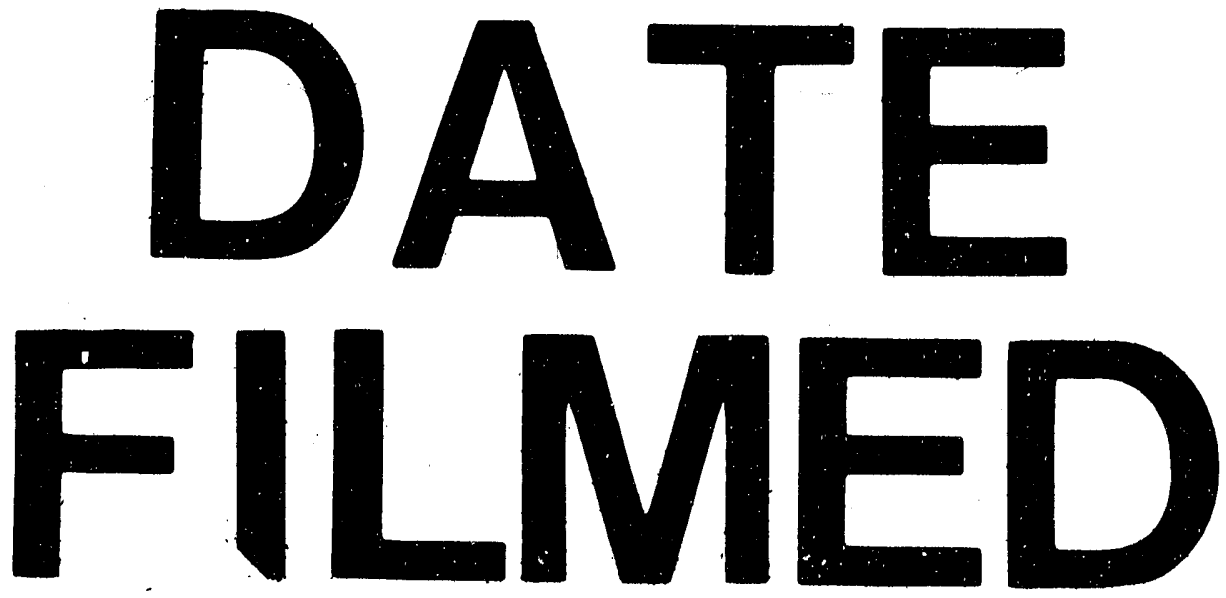

$8 / 17 / 92$ 